\title{
A novel germline mutation of PTEN associated with brain tumours of multiple lineages
}

\section{FJT Staal*,', RB van der Luijt ${ }^{2}$, MRM Baert', J van Drunen², H van Bakel ${ }^{2}$, E Peters' ${ }^{2}$ I de Valk $^{2}$, HKP van Amstel ${ }^{2}$, MJB Taphoorn ${ }^{3}$, GH Jansen ${ }^{4}$, CWM van Veelen ${ }^{3}$, B Burgering ${ }^{5}$ and GEJ Staal ${ }^{2}$}

\begin{abstract}
'Department of Immunology, Erasmus University Rotterdam, Rotterdam, the Netherlands; '2Department of Medical Genetics, University Medical Center, Utrecht, the Netherlands; ${ }^{3}$ Department of Neuro-oncology and Neurosurgery, University Medical Center, Utrecht, the Netherlands; ${ }^{4}$ Department of Pathology, University Medical Center, Utrecht, the Netherlands; ${ }^{5}$ Department of Physiological Chemistry, University Medical Center, Utrecht, the Netherlands
\end{abstract}

PTEN, also designated as MMAC1 or TEP1, is a tumour suppressor gene located on chromosome 10q23 (Li et al, 1997; Bonneau and Longy, 2000). Somatic PTEN mutations have been identified in a variety of human cancers including breast, prostate, thyroid and endometrial carcinomas, malignant melanomas and gliomas (Bonneau and Longy, 2000). Mutation of PTEN appears to occur early in tumour development in endometrial cancer but late in the development of gliomas. Germline mutations in PTEN have also been found in the dominantly inherited Cowden, LhermitteDuclos, and Bannayan-Zonana syndromes, which are characterised by the formation of multiple benign tumours (Nelen et al, 1997).

Disruption of PTEN in the mouse results in early embryonic lethality. PTEN heterozygous mice display hyperplastic features as well as high tumour incidence suggesting that inactivation of PTEN plays important roles in tumorigenicity (Di Cristofano et al, 1998; Suzuki et al, 1998; Podsypanina et al, 1999). The PTEN gene contains nine exons and encodes a 403-aa protein, which possesses phosphatase activity on phosphotyrosyl and phosphoseryl/threonyl residues (Li and Sun, 1997; Bonneau and Longy, 2000). PTEN is an inefficient protein phosphatase in vitro but is very active on highly acidic substrates. The main PTEN substrate has proven to be phosphatidyl inositol $(3,4,5)$-triphosphate (PIP-3), which is the direct product of PI-3-kinase (Maehama and Dixon, 1998).

*Correspondence: Dr FJT Staal; Department of Immunology, Erasmus University Rotterdam, Dr. Molewaterplein 50, 3015 GE Rotterdam, the Netherlands; E-mail: f.j.t.staal@immu.fgg.eur.nl

Received I5 October 200I; revised 7 January 2002; accepted 24 January 2002
It has been proven that cells lacking wild-type PTEN from gliomas are characterised by elevated levels of PIP-3 (Myers and Tonks, 1997). As a consequence, the activity of protein kinase B (PKB/Akt) was found to be elevated, resulting in prevention of apoptosis (Stambolic et al, 1998). Introduction of wild-type PTEN reduced the levels of PIP-3 and protein kinase B ( $\mathrm{Li}$ and Sun, 1998). In addition, wild-type PTEN was found to suppress the proliferation and the tumour growth of PTEN deficient glioblastoma cells, whereas mutant phosphatase inactive PTEN failed to suppress cell growth (Cheney et al, 1998; Furnari et al, 1998). Thus, two biological processes, namely cell proliferation and apoptosis are partly regulated by PTEN activity.

In searching for PTEN mutations in human gliomas we detected a heterozygous mutation (Arg234Gln) of PTEN in a patient with a glioma. The mutation was present in tumour DNA, as well as in DNA isolated from peripheral blood lymphocytes. No signs of Cowden disease were found in the patient, nor did we find any indications for loss of heterozygosity (LOH) of PTEN in tumour material. The biological consequences of this germline mutation have been evaluated by means of transfection studies of the mutant PTEN molecule compared to wild-type PTEN. Our results suggest that this mutation plays a role in regulating apoptosis and cell proliferation.

\section{PATIENT AND METHODS}

\section{Patient and histology}

In 1981 a 38-year old male, with an unremarkable medical history and with no family history of brain tumours, presented with focal seizures of the right arm and dysphasia. No neurological abnorm- 
alities were found on examination. Anti-epileptic drug medication resulted in a marked reduction of the seizures. In 1985 a CT brain scan was made because of progressive headaches and memory difficulties. A large right frontal lesion was found suggestive of a meningioma; this was removed completely. The diagnosis of meningioma was confirmed by the pathologist, but there were no signs of malignancy. In 1990 the seizures spread to the patient's right leg.

A CT scan revealed a non-enhancing lesion with slight mass effect in the left frontal lobe; a low grade glioma was suspected. The patient's symptoms progressed and he underwent surgery for this lesion in 1993. Histologic examination revealed tumour cells with peri-nuclear unstained cytoplasm, with irregular round to oval nuclei, with some mitotic activity (three per square $\mathrm{mm}$ ). The capillary network in the tumour was unremarkable and there was no necrosis. This tumour was classified as an anaplastic oligodendroglioma. The patient underwent radiotherapy.

In 1998 the patient suffered a decline of cognitive functioning. A new CT brain scan showed regrowth of the left frontal tumour, which was again treated surgically. Histologic examination revealed a similar profile to that in 1993, but with more pleiomorphism of the nuclei of the tumour cells, more mitotic activity (eight per square $\mathrm{mm}$ ), microvascular proliferation, and small areas of necrosis. The diagnosis was an anaplastic oligodendroglioma with signs of ongoing de-differentiation.

He was treated with chemotherapy for residual tumour, but after nearly a year there was further tumour growth. His clinical condition is steadily deteriorating.

\section{Tumour sample}

Tumour samples were collected in the Department of Neurosurgery, University Medical Center, Utrecht, the Netherlands. Immediately after removal, parts of the tumour were snap-frozen in liquid nitrogen and stored at $-80^{\circ} \mathrm{C}$ until required.

\section{DNA extraction}

Tumour tissue was cut into $10 \mu \mathrm{m}$ thick sections and mounted onto glass slides. Reference slides were stained with haematoxylin and eosin and examined by the pathologist in order to grade the tumour according to WHO criteria and for selecting tumour regions for analysis. The marked slide was used to guide microdissections. DNA extraction was carried out with the use of proteinase $\mathrm{K}$ and phenol/chloroform. High molecular weight DNA of the patient was also isolated from peripheral blood according to established procedures.

\section{LOH analysis}

For LOH analysis, a total of six highly polymorphic markers from the PTEN region were used: five microsatellite markers flanking PTEN (D10S1645，D10S579，D10S215，D10S603 and D10S541) and the intragenic marker D10S2491. PCR reactions were performed in a GeneAmp 9600 PCR System (PE Biosystems) in a $10 \mu \mathrm{l}$ volume containing $30 \mathrm{ng}$ genomic DNA as template, $25 \mathrm{ng}$ of each oligonucleotide primer, $200 \mu \mathrm{M}$ of each dNTP and 0.4 unit AmpliTaq Gold DNA polymerase (Perkin Elmer), in $1 \times$ AmpliTaq Gold buffer with $2.5 \mathrm{~mm} \mathrm{MgCl}_{2}$. An initial denaturation of $5 \mathrm{~min}$ at $95^{\circ} \mathrm{C}$ was followed by 36 cycles of $30 \mathrm{~s}$ at $95^{\circ} \mathrm{C}, 30 \mathrm{~s}$ at $55^{\circ} \mathrm{C}, 30 \mathrm{~s}$ at $72^{\circ} \mathrm{C}$, and a final extension step of $10 \mathrm{~min}$ at $72^{\circ} \mathrm{C}$. Markers were analysed on an ABI Prism ${ }^{\mathrm{TM}} 377$ DNA Sequencer (PE Biosystems) using GeneScan and Genotyper software.

\section{Sequence analysis}

Direct sequencing of the nine PTEN exons and their immediately flanking regions was performed using the oligonucleotide primers published by Steck et al (1997). PCR reactions were performed in a GeneAmp 9600 PCR System (PE Biosystems) in a $50 \mu \mathrm{l}$ volume containing $100 \mathrm{ng}$ genomic DNA as template, $50 \mathrm{ng}$ of each oligonucleotide primer, $200 \mu \mathrm{M}$ of each dNTP and 1.25 unit AmpliTaq Gold DNA polymerase (Perkin Elmer), in $1 \times$ AmpliTaq Gold buffer with $2.5 \mathrm{~mm} \mathrm{MgCl} 2$. An initial denaturation of $10 \mathrm{~min}$ at $95^{\circ} \mathrm{C}$ was followed by 35 cycles of $30 \mathrm{~s}$ at $95^{\circ} \mathrm{C}, 30 \mathrm{~s}$ at $55^{\circ} \mathrm{C}$, $1 \mathrm{~min}$ at $72^{\circ} \mathrm{C}$, and a final extension step of $10 \mathrm{~min}$ at $72^{\circ} \mathrm{C}$. For the amplification of exons 1 and 2, the annealing temperature was 57 and $50^{\circ} \mathrm{C}$, respectively. Sequencing was performed on an ABI Prism ${ }^{\mathrm{TM}} 377$ DNA Sequencer (PE Biosystems) using the Rhodamine Terminator Cycle Sequencing Ready Reaction Kit, according to the manufacturer's instructions.

\section{Constructs, transfections and proliferation assays}

Wild-type and Arg234Gln mutant PTEN were cloned into the pCDNA3 expression vector by using PCR primers containing restriction sites. For wild-type PTEN the forward primer contained a BamHI site, the reverse primer a XhoI site. The mutant site was introduced by means of two partially overlapping primers containing the mutation and introducing an EcoRI site. The EcoRI site was used to confirm the presence of the mutation in the final construct.

Sequences: PTEN FW: 5' CAAGGATCCACCATGACAGCCATCATCAAAGAG $3^{\prime}$

PTEN RV: $5^{\prime}$ CGTTGCCTCGAGTCAGACTTTTGTAATTTGTGTATG $3^{\prime}$

MUT PTEN FW: 5' GCCTGAATTCGAGGAATATATCTTCACCTTTAG $3^{\prime}$

MUT PTEN RV: $5^{\prime}$ TTCCGAATTCAGGACCCACACGACAGGAAGACAAGTTCATGTACTTC 3'.

PCR was performed in a $50 \mu \mathrm{l}$ volume containing $100 \mathrm{ng}$ pSG5PTENwt as template, $50 \mathrm{ng}$ of each oligonucleotide primer, $200 \mu \mathrm{M}$ of each dNTP and 1.25 unit AmpliTaq Gold DNA polymerase (Perkin Elmer), in $1 \times$ AmpliTaq Gold buffer with $2.5 \mathrm{mM} \mathrm{MgCl}_{2}$. An initial denaturation of $10 \mathrm{~min}$ at $95^{\circ} \mathrm{C}$ was followed by 25 cycles of $30 \mathrm{~s}$ at $95^{\circ} \mathrm{C}, 30 \mathrm{~s}$ at $57^{\circ} \mathrm{C}, 1.25 \mathrm{~min}$ at $72^{\circ} \mathrm{C}$, and a final extension step of $7 \mathrm{~min}$ at $72^{\circ} \mathrm{C}$. PCR products were separated on a $1 \%$ agarose gel, purified, and digested with $\mathrm{XhoI}$ and BamHI (wt), or the two PCR products for mutant PTEN with BamHI and EcoRI and with EcoRI and XhoI. Wt PTEN was cloned into BamHI/XhoI digested pCDNA3 by direct ligation, and the mutant PTEN by three-point ligation. Integrity of the wild-type and mutant forms was confirmed by sequencing the full inserts, as above. For transfections, the plasmids were digested with $X b a \mathrm{I}$, in order to linearise them outside the neomycin resistance cassette and outside the PTEN coding sequence.

U87 glioma cells, which lack functional PTEN were transfected with the pcDNA expression constructs (which contains a neomycin resistance gene) using Fugene-6 (Boehringer Mannheim), according to the manufacturer's instructions. Two days after transfection, cells were put under selection using G418 $(1.0 \mathrm{mg} / \mathrm{ml})$. After approximately 3 weeks of selection, stable selected cells were checked for cell proliferation by seeding 5000 cells from the stable transfectants and manual counting at days $4,6,8$ and 11 .

\section{Western blotting}

Equal numbers of U87 cells were lysed in boiling SDS sample buffer, loaded on a $10 \%$ polyacrylamide gel, separated by electrophoresis and blotted onto nitrocellulose by standard methods. PTEN protein was revealed using the anti-PTEN Antibody 9522 (Cell signalling Technology) and a Goat anti-rabbit-HPRO second step antibody, followed by visualisation by enhanced chemiluminescence (ECL, Amersham). For PKB assays, U87MG cells were seeded and grown to subconfluency, serum-starved for $18 \mathrm{~h}$, and either left untreated or treated with insulin for $10 \mathrm{~min}$ then washed with PBS twice and directly harvested in Leamlli sample buffer. 
Fifty $\mu \mathrm{g}$ of protein was analysed by SDS-PAGE for phosphorylation of $\mathrm{PKB}$ by immunoblotting using an antibody against phosphorylated serine 473 . The phosphorylation of this residue activates $\mathrm{PKB} /$ Akt.

\section{Apoptosis assay}

Expression plasmids that target PTEN to the cell membrane using the CAAX motif were constructed in pcDNA by standard methods. Apoptosis assays were done as described (Kalejta et al, 1997). The CAAX constructs or empty vector $(18 \mu \mathrm{g})$ were transfected by electroporation $(270 \mathrm{~V} ; 960 \mathrm{uF})$ into $\mathrm{BaF} 3$ promyelocytic cells, together with a Spectrin-GFP plasmid $(2 \mu \mathrm{g})$. Two hours after transfection viable cells were collected by Ficoll density centrifugation, and cultured for another $24 \mathrm{~h}$. The next day cells were harvested and resuspended in $300 \mu \mathrm{l}$ PBS to which $700 \mu \mathrm{l}$ ice cold $100 \%$ ethanol was added. Cells were incubated at $-20^{\circ} \mathrm{C}$ for $2 \mathrm{~h}$, washed and incubated with a propidium iodide/RNAse solution for $30 \mathrm{~min}$ at $37^{\circ} \mathrm{C}$ and put on ice in the dark till analysis on a FACScan flow cytometer. The number of transfected apoptotic cells was enumerated by electronic gating on GFP+ cells, and generating a cell cycle profile for these cells. The percentage of sub $2 \mathrm{n}$ cells was taken as a measure for the percentage of apoptosis.

\section{RESULTS}

\section{Mutation analysis and LOH}

The PTEN gene from a patient with glioma was analysed. PCR of all nine exons of the PTEN gene followed by sequencing revealed a $\mathrm{G}$ to $\mathrm{A}$ (CGACGG $\rightarrow$ CGACAGG) substitution at position 701 (PTEN cDNA sequence U93051, GenBank) in exon 7, changing an arginine (Arg) residue to a glutamine (Gln) at codon 234. Figure 1 depicts the sequence analysis showing the missense mutation detected. The Arg234Gln (R234Q) mutation is located at the surface of the protein of the so-called C-2 domain (Lee, 1999). The mutation was found in DNA obtained from peripheral blood, the meningioma and the two oligodendrogliomas of the patient.

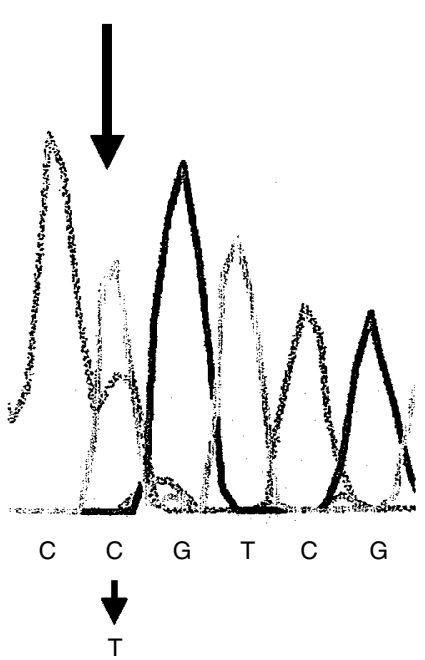

Figure I Identification of an Arg234GIn germline PTEN mutation in a patient with multiple brain tumours. A partial sequence of exon 7, obtained from tumour (glioma) DNA is shown. In the sequence (which is in reverse orientation), the single base change ( $\mathrm{C}$ to $\mathrm{T}$ ) is indicated by an arrow. The heterozygous peak corresponds to a $\mathrm{G}$ to $\mathrm{A}$ substitution at position 70 I in the PTEN coding sequence, and results in an arginine to glutamine amino acid change at codon 234. Note the heterozygous state of the missense mutation, suggesting that there is no $\mathrm{LOH}$ of PTEN in the tumour.
The mutation has not been observed in 80 normal control chromosomes (data not shown), therefore it is unlikely to represent a common polymorphism.

Taken together, this therefore indicates that the R234Q mutation is a pathogenic germline mutation. No family studies could be carried out. To determine whether the PTEN mutation detected was associated with loss of heterozygosity in chromosome $10 \mathrm{q}$, microsatellite analysis was carried out using markers mentioned in Materials and Methods. No LOH could be detected, which is in agreement with the heterozygous nature of the mutation in tumour material observed by DNA sequencing.

\section{Cell proliferation analysis}

In order to functionally characterise the consequence of the R234Q mutation identified, we performed two different assays concerning two processes, in which PTEN has been shown to play a key role, namely cell proliferation and apoptosis. We cloned wild-type and mutant PTEN into an expression plasmid and stably transfected U87MG glioma cells that lack functional PTEN. In this cell line, the PTEN gene carries a mutation in the splice donor site of intron 3, resulting in deletion of exon 3 in the mature transcript, leading to an unstable, nonfunctional protein that can only be detected at low levels. Western blot analysis revealed that PTEN levels in the transfectants were roughly equal to each other (Figure 2) and similar to cell lines that contain functional PTEN (not shown). In contrast, the U87 mother cell line contains lower levels of (non-functional) PTEN compared to the transfectants (Figure 2).

The wild-type and mutant PTEN transfectants were compared for their growth properties. By counting the number of cells over a 2-week period, it became apparent that the mutant cells proliferated at a roughly five-fold higher rate than cells transfected with wild-type PTEN (Figure 3). Thus, the R234Q mutation in PTEN that we found permits cells to proliferate at a much higher rate.

\section{Apoptosis}

The wild-type and mutant PTEN were cloned into a so-called CAAX vector to target them to the cell membrane. Using BaF3 promyelocytic cells, which are IL-3 dependent and sensitive to apoptosis, we investigated the consequence of these forms of PTEN for apoptosis. In order to identify transfected cells, the PTEN molecules were co-transfected with a spectrin-GFP construct. Gating on GFP positive cells thus permitted investigating only transfected cells. As shown in Figure 4, cells transfected with an empty vector (together with Spectrin-GFP), have approximately 18\% apoptotic cells. Transfection of wild-type PTEN almost doubles the percentage of apoptotic cells $(32 \%)$, in contrast mutant PTEN does not induce any apoptosis ( $16 \%$ of sub $2 \mathrm{n}$ cells) and in some experiments the percentage of apoptotis after transfection of the mutant was even lower than in cells transfected with SpectrinGFP alone.

\section{PKB/Akt activity}

U87MG cells stably expressing either wild-type PTEN or the R234Q mutant were compared with respect to the phosphorylation status of PKB. Phosphorylation of PKB at threonine 308 and serine 473 is indicative for the activity of $\mathrm{PKB}$ (reviewed in Chan et al, 1999). As reported previously ((Maier et al, 1999) and data not shown) parental U87MG cells displayed elevated levels of PKB phosphorylation which could still be further elevated by treatment of cells with insulin for $10 \mathrm{~min}$ (see Figure 5). Stable expression of wild-type PTEN did not significantly alter PKB regulation. In contrast however, expression of the $\mathrm{R} 234 \mathrm{Q}$ mutant resulted in a further increase in $\mathrm{PKB}$ phosphorylation that could not be induced further by insulin treatment. 


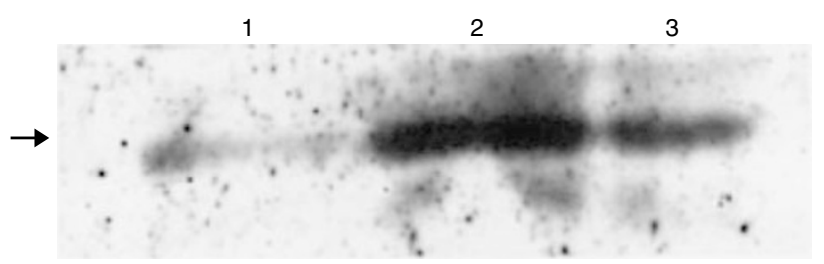

Figure 2 Western blot of stably transfected U87-MG glioma cells. The U87 glioma cell has a dysfunctional PTEN molecule and was transfected with R234Q mutant (lane 2) or wild-type (lane I). Stable transfectants express higher levels of PTEN than the parental line (lane 3). The $54 \mathrm{kD}$ PTEN band is indicated by an arrow.

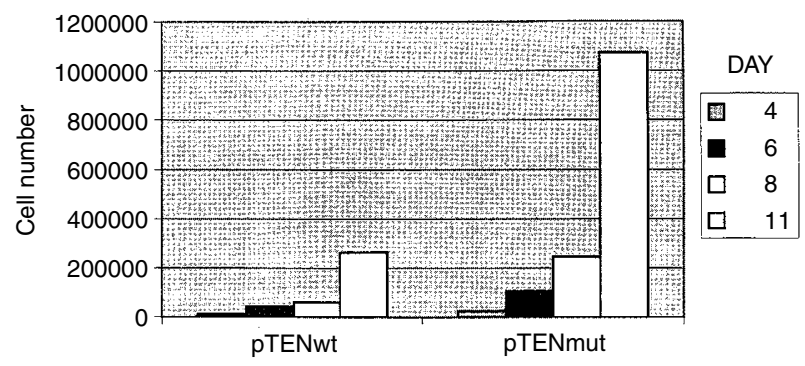

U87

Figure 3 R234Q Mutant PTEN allows higher levels of proliferation than wild-type PTEN. The stable U87 transfectants were grown under neo-selection and 5000 cells were seeded on day 0 . Pints cells were harvested and counted at the indicated time.

\section{DISCUSSION}

Here we report on a novel PTEN mutation that was found as a germline mutation in a patient with meningioma and glioma. To our knowledge this is the first germline mutation reported for brain tumours of multiple lineages. The mutation changes an arginine residue to a glutamine in the $\mathrm{C}-2$ domain of PTEN. This changes the charge of that region (from positive to neutral). Functions of the C-2 region may involve membrane targeting through interactions with membrane phospholipids or association with as yet unknown proteins (Lee, 1999). It has also been shown that the C-2 domain functions to position the phosphatase domain correctly with respect to its substrates. Therefore, mutations in the C-2 domain could alter the overall enzymatic activity of PTEN, without necessarily affecting its phosphatase activity.

We demonstrate that this mutation has functional consequences because the mutant molecules allow cells to proliferate at much higher levels and do not induce or prevent apoptosis. Given that mice with a targeted PTEN mutation on one allele and a wild-type PTEN molecule on the other allele show abnormalities (Di Cristofano et al, 1998, 1999), the heterozygous nature of the mutation in the patient does not diminish the importance of the functional findings. The functional consequences of the mutation we have found may help explain why the tumour arose. Of course, it is possible that this mutation has nothing to do with the development of the tumour, or that this is a syndrome with a very low penetrance. However, it seems likely that this mutation also has functional consequences in the tumour. Competition for limited substrates or associated molecules may interrupt normal PTEN function sufficiently to lead to increased proliferation and diminished apoptosis. In fact, the mutant molecule is incapable of inducing apoptosis in $\mathrm{BaF} / 3$ cells, which contain endogenous functional PTEN. In some experiments the percentage of apoptotic cells after transfection of R234Q PTEN was even lower than GFP alone, perhaps because endogenous PTEN activity was somewhat inhibited. Another indication that this mutation may not be solely a
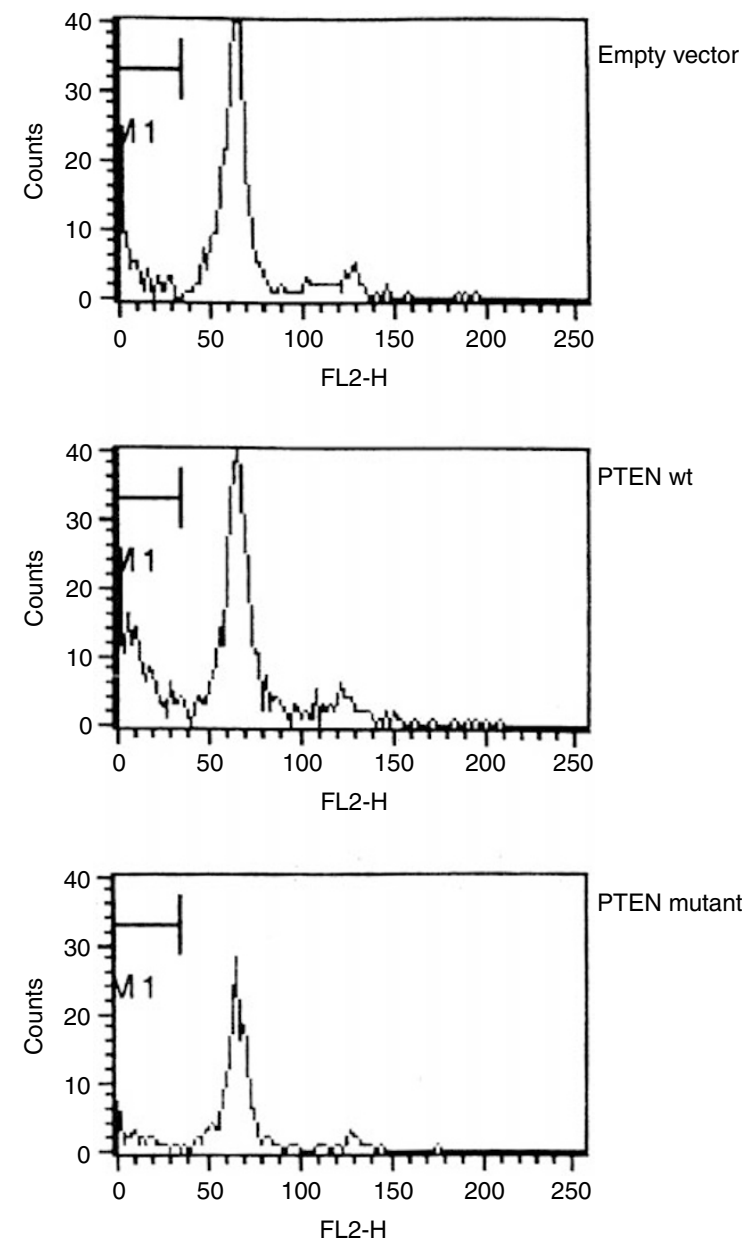

Figure 4 Mutant PTEN does not induce apoptosis, whereas wild-type does. BAF/3 cells were transfected with Spectrin-GFP, together with empty vector control (A) CAAX-PTEN wild-type (B), CAAX-PTEN mutant (C). Cell cycle profiles for propidium iodide stained cells, electronically gated on GFP+ cells are shown.

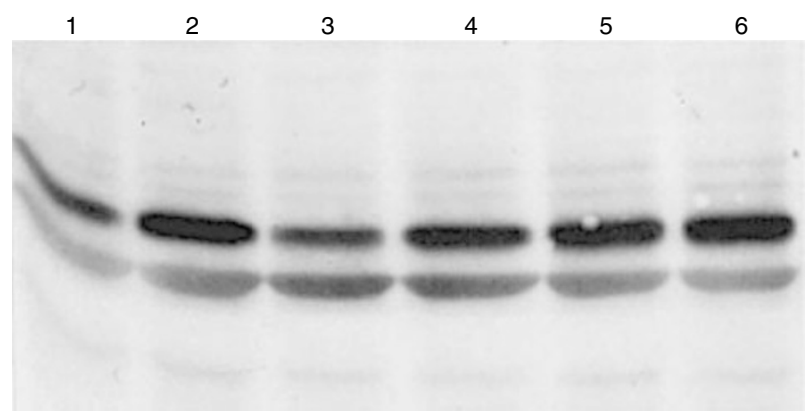

Figure 5 Increased constitutive PKB activity, but lack of insulin-induced PKB activity in R234Q PTEN transfectants. U87MG transfected wild-type cells (lanes I and 2), U87MG cells transfected with normal PTEN (lanes 3 and 4 ) and mutant PTEN (lanes 5 and 6 ) were seeded and grown to subconfluency, serum-starved for $18 \mathrm{~h}$ and either left untreated (lanes I, 3, 5) or treated with insulin (lanes 2, 4,6) for 10 min then washed with PBS twice and directly harvested in Leamlli sample buffer. Fifty $\mu \mathrm{g}$ of protein were analysed by SDS - PAGE for phosphorylation of PKB by immunoblotting using an antibody against phosphorylated serine 473. The phosphorylation of this residue activates PKB/Akt. The transfection of wild-type PTEN has little effect, whereas the R234Q mutant induces constitutive phosphorylation of PKB, which cannot be increased further by insulin treatment. 
PTEN null mutant, but can act as a negative competitor, stems from experiments investigating $\mathrm{PKB}$ activity. In R234Q PTEN transfectants, PKB activity could not be induced by insulin treatment, in contrast to parental cells or cells transfected with wt PTEN. This result suggests that the R234Q mutation acts to inhibit the residual PTEN activity present in U87MG cells and can therefore be considered as acting as a negative competitor. Thus, the functional consequences of the mutation in transfection studies are consistent with high proliferative activity. Together, these findings suggest that the Arg234Gln missense mutation in PTEN has oncogenic properties.

The main PTEN substrate has proved to be phosphatidyl inositol (3,4,5)-triphosphate (PIP-3) (Myers and Tonks, 1997). Previously, it has been shown that glioma cells lacking wild-type PTEN are characterised by elevated levels of PIP-3 (Li and Sun, 1998). As a consequence, the activity of protein kinase B (PKB/ Akt) was found to be elevated, resulting in prevention of apoptosis and increased proliferation. Our results fit this picture in that U87MG cells stably transfected with the R234Q mutant PTEN also show increased proliferation and increased constitutive PKB activity.

One of the main functions of PTEN is to keep PIP-3 levels low. Loss of PTEN function results in increased PIP-3 levels, leading to hyperactive $\mathrm{PKB} / \mathrm{Akt}$. The mutation we have found may similarly affect PTEN activity, finally resulting in increased PKB activity, increased cell proliferation, and diminished apoptosis.

Not many PTEN mutations described in the literature have been associated with the C-2 domain, although Lee et al (1999) reported that mutagenesis of the C-2 domain reduced the tumour suppressive activity of PTEN. Our findings are consistent with these results. It is tempting to speculate, based on the X-ray crystallography model of PTEN (see Figure 6), that the C-2 loop can associate with other proteins. Interestingly the mutation is exactly in the middle of the loop, at a site readily accessible for interaction with other molecules. Future research will undoubtedly identify such C-2 domain-interacting molecules, most likely further explaining the oncogenic nature of the PTEN mutation we have described here. It has been proposed that mutation of basic residues in the $\mathrm{C}-2$ domain reduces the membrane affinity of PTEN and its ability to suppress the growth of glioma tumour cells (Lee et al, 1999).

Germline mutations of the PTEN gene are responsible for several inherited conditions, including Cowden disease, Bannayan-Zonana syndrome and Lhermitte-Duclos disease. Here we present a patient with a heterozygous PTEN germline mutation but with no signs of any of these disorders. The patient had an oligodendroglioma, and, in the past, a benign meningioma. The PTEN gene is a tumour suppressor gene and inactivation of both alleles is required for neoplastic transformation. An inherited

\section{REFERENCES}

Bonneau D, Longy M (2000) Mutations of the human PTEN gene. Hum Mutat 16: $109-122$

Chan TO, Rittenhouse SE, Tsichlis PN (1999) AKT/PKB and other D3 phosphoinositide-regulated kinases: kinase activation by phosphoinositidedependent phosphorylation. Annu Rev Biochem 68: 965-1014

Cheney IW, Johnson DE, Vaillancourt MT, Avanzini J, Morimoto A, Demers GW, Wills KN, Shabram PW, Bolen JB, Tavtigian SV, Bookstein R (1998) Suppression of tumorigenicity of glioblastoma cells by adenovirusmediated MMAC1/PTEN gene transfer. Cancer Res 58: 2331-2334

Di Cristofano A, Pesce B, Cordon-Cardo C, Pandolfi PP (1998) Pten is essential for embryonic development and tumour suppression. Nat Genet 19: $348-355$

Di Cristofano A, Kotsi P, Peng YF, Cordon-Cardo C, Elkon KB, Pandolfi PP (1999) Impaired Fas response and autoimmunity in Pten $+/-$ mice. Science 285: $2122-2125$

Furnari FB, Huang HJ, Cavenee WK (1998) The phosphoinositol phosphatase activity of PTEN mediates a serum-sensitive G1 growth arrest in glioma cells. Cancer Res 58: $5002-5008$

Kalejta RF, Shenk T, Beavis AJ (1997) Use of a membrane-localized green fluorescent protein allows simultaneous identification of transfected cells and cell cycle analysis by flow cytometry. Cytometry 4: 286-291

Lee JO, Yang H, Georgescu MM, Di Cristofano A, Maehama T, Shi Y, Dixon JE, Pandolfi P, Pavletich NP (1999) Crystal structure of the PTEN tumor suppressor: implications for its phosphoinositide phosphatase activity and membrane association. Cell 99: $323-334$

Li DM, Sun H (1997) TEP1, encoded by a candidate tumor suppressor locus, is a novel protein tyrosine phosphatase regulated by transforming growth factor beta. Cancer Res 57: 2124-2129 
Li J, Yen C, Liaw D, Podsypanina K, Bose S, Wang SI, Puc J, Miliaresis C, Rodgers L, McCombie R, Bigner SH, Giovanella BC, Ittmann M, Tycko B, Hibshoosh H, Wigler MH, Parsons R (1997) PTEN, a putative protein tyrosine phosphatase gene mutated in human brain, breast, and prostate cancer. Science 275: $1943-1947$

Li DM, Sun H (1998) PTEN/MMAC1/TEP1 suppresses the tumorigenicity and induces G1 cell cycle arrest in human glioblastoma cells. Proc Natl Acad Sci USA 95: 15406-15411

Maehama T, Dixon JE (1998) The tumor suppressor, PTEN/MMAC1, dephosphorylates the lipid second messenger, phosphatidylinositol 3,4,5trisphosphate. J Biol Chem 273: 13375-13378

Maier D, Jones G, Li X, Schonthal AH, Gratzl O, Van Meir EG, Merlo A (1999) The PTEN lipid phosphatase domain is not required to inhibit invasion of glioma cells. Cancer Res 59: $5479-5482$

Myers MP, Tonks NK (1997) PTEN: sometimes taking it off can be better than putting it on [editorial]. Am J Hum Genet 61: 1234-1238

Nelen MR, van Staveren WC, Peeters EA, Hassel MB, Gorlin RJ, Hamm H, Lindboe CF, Fryns JP, Sijmons RH, Woods DG, Mariman EC, Padberg GW, Kremer H (1997) Germline mutations in the PTEN/MMAC1 gene in patients with Cowden disease. Hum Mol Genet 6: 1383-1387
Podsypanina K, Ellenson LH, Nemes A, Gu J, Tamura M, Yamada KM, Cordon-Cardo C, Catoretti G, Fisher PE, Parsons R (1999) Mutation of Pten/Mmacl in mice causes neoplasia in multiple organ systems. Proc Natl Acad Sci USA 96: 1563-1568

Stambolic V, Suzuki A, de la Pompa JL, Brothers GM, Mirtsos C, Sasaki T, Ruland J, Penninger JM, Siderovski DP, Mak TW (1998) Negative regulation of $\mathrm{PKB} / \mathrm{Akt}$-dependent cell survival by the tumor suppressor PTEN. Cell 95: 29-39

Steck PA, Pershouse MA, Jasser SA, Yung WK, Lin H, Ligon AH, Langford LA, Baumgard ML, Hattier T, Davis T, Frye C, Hu R, Swedlund B, Teng DH, Tavtigian SV (1997) Identification of a candidate tumour suppressor gene, MMAC1, at chromosome 10q23.3 that is mutated in multiple advanced cancers. Nat. Genet. 4: $356-362$

Suzuki A, de la Pompa JL, Stambolic V, Elia AJ, Sasaki T, del Barco, Barrantes I, Ho A, Wakeham A, Itie A, Khoo W, Fukumoto M, Mak TW (1998) High cancer susceptibility and embryonic lethality associated with mutation of the PTEN tumor suppressor gene in mice. Curr Biol 8: 1169-1178 